\title{
La representación social que orienta las decisiones paternas al afrontar el consumo de drogas de sus hijos
}

Bertha Lidia N uño-Gutiérrez, M en Terap Fam Sist, ${ }^{(1)}$ Catalina González-Forteza, Dra en Psic Soc.(2)

\section{Nuño-Gutiérrez BL, González-Forteza C. La representación social que orienta las decisiones paternas al afrontar el consumo de drogas de sus hijos.} Salud Publica Mex 2004;46:123-131. El texto completo en inglés de este artículo está disponible en: http://www.insp.mx/salud/index.html

\section{Resumen}

Objetivo. Identificar la representación social que orientó la toma de decisiones en padres de adolescentes usuarios de dro gas para afrontar el consumo de sus hijos. Material y métodos Por selección aleatoria sistemática se realizó un estudio cualitativo mediante listados libres, cuestionarios de caracterización y entrevistas profundas a 60 padres de usuarios que acudían a tratamiento a Centros de Integración Juvenil en G uadalajara, Jalisco, México, dur ante 2002. El análisis fue mediante correlaciones y análisis interpretativo. Resultados. Se identificaron tres etapas:1) descubriendo la adicción, caracterizada por decepción de los padres; 2) permanencia: la más prolongada en tiempo, inversión de recursos y pérdidas, y 3) retirada: caracterizada por debut en ser vicios de salud. Conclusiones La representación que orientó las diferentes decisiones fue la oferta de un mundo mejor, la cual emergió del contexto social, cultural y familiar que gira alrededor del consumo, y fue mantenida por dos creencias: "aprendiendo de los errores" y "querer es poder". El texto completo en inglés de este artículo está disponible en: http://www.insp.mx/salud/index.html

Palabras clave: adicción; adolescencia; dro gas; familia; representaciones sociales; ter apia familiar; M éxico

\author{
Nuño-Gutiérrez BL, González-Forteza C. \\ The social representation guiding \\ parental decisions to face drug consumption \\ of their teenage children. \\ Salud Publica Mex 2004;46:123-131. \\ The English version of this paper \\ is available at: http://www.insp.mx/salud/index.html
}

\begin{abstract}
A bstract
Objective To identify the social representation guiding decisions of parents of teenage drug users to face consumption of their children. Material and Methods. A qualitative study using free lists, characterization questionnaires, and in-depth interviews was performed among 60 systematically selected parents of young drug users who were receiving treatment in Centros de Integración Juvenil. $\mathrm{D}$ ata analysis included correlations and interpretive analysis. Results. Three stages were identified: 1) discovery of the addiction, characterized by parental disappointment; 2) permanence:The highest in duration, resource investment, and losses; and 3) withdrawal: Featured by seeking health services. Conclusions. The representation that guided parental decisions was "the offer of a better world", which stemmed from the social, cultural, and family context that revolves around consumption and that was sustained by two beliefs: "Learning from mistakes" and "where there is a will there is a way". The English version of this paper is available at: http://www.insp.mx/salud/index.html
\end{abstract}

Key words: addiction; adolescence; drugs; family; family therapy; social representations; Mexico

Proyecto financiado por el Fondo de Fomento a la Investigación, del Instituto Mexicano del Seguro Social.

(1) Unidad de Investigación Epidemiológica y en Servicios de Salud del Adolescente. Instituto Mexicano del Seguro Social. Delegación Jalisco. Tonalá, Jalisco, México.

(2) Departamento de Investigaciones en Servicios de Salud, D irección de Investigaciones Epidemiológicas y Psicosociales. Instituto N acional de Psiquiatría Ramón de la Fuente Muñiz. México, DF, México.

Fecha de recibido: 7 de noviembre de 2003 - Fecha de aprobado: 21 de enero de 2004 Solicitud de sobretiros: Bertha Lidia N uño G utiérrez, Avenida Tonalá No 121, 45400.Tonalá, Jalisco, México. Correo electrónico: bnuno@ udgserv.cencar.udg.mx 
L teoría de las representaciones sociales surgió en la psicología francesa, con la publicación La psychanalise son image et son public de Serge Moscovici, en 1961. ${ }^{1}$ Moscovici entiende las representaciones sociales (RS) como "entidades casi tangibles [que] circulan, se cruzan y se cristalizan sin cesar en nuestro universo cotidiano a través de una palabra, un gesto, un encuentro. La mayor parte de las relaciones sociales [...] de los objetos producidos o consumidos en las comunicaciones intercambiadas están impregnadas de ellas". 2

Moscovici ${ }^{2}$ afirma que para calificar de social a una representación no es suficiente saber quién la produce, sino por qué la produce. En la literatura ${ }^{2-5}$ se identifican cuatro funciones: a) del saber, que permiten entender y explicar la realidad; b) las identitarias, que sitúan a los individuos y grupos en el campo social y le permiten elaborar una identidad; c) de orientación, que prescriben comportamientos y prácticas, y d) justificadoras, que permiten justificar a posteriori posturas y comportamientos.

La finalidad primordial de las RS estriba en que los individuos se explican, modelan y remodelan su realidad a través de sus prácticas sociales que son mediadas por las decisiones. De acuerdo con Moscovici, ${ }^{2}$ las tomas de decisión no son productos arbitrarios, sino razonados, que subyacen en los conocimientos y sistemas de creencias que giran alrededor del objeto. Estos razonamientos no son producto de teorías elaboradas, sino que provienen del sentido común con el cual los individuos operan en su vida cotidiana. Esto es a lo que se refería Moscovici cuando introdujo el término de sociedad pensante, ${ }^{2}$ al sostener que los individuos intentan resolver sus problemas cotidianos utilizando las RS que crean y comparten a través de la interacción comunicacional.

Dicho de otra forma, las RS son formas de pensamiento social que incluyen implícita y explícitamente informaciones, creencias, actitudes, prácticas y experiencias, que orientan a posteriori la toma de decisiones. ${ }^{2-6}$

Por otro lado, la noción de modelos de toma de decisiones tuvo sus antecedentes en Kuhn, ${ }^{7}$ cuando propuso cómo operaba un paradigma. En la terapia familiar se ha usado para referirse a cómo las personas le atribuyen cualidades al mundo social, que no son sometidas a discusión por su adherencia ideológica. ${ }^{8,9}$ Estos modelos fueron definidos por De Shazer ${ }^{10}$ como un conjunto constituyente de ideas que operan como paradigmas culturales bajo los cuales se construyen problemas y soluciones. Su objetivo es identificar las reglas que los rigen para planificar la intervención terapéutica. ${ }^{10-15}$ Sin embargo, estos modelos tienen deficiencias: no interesa el tipo de problema, su evolución, las experiencias, la cultura histórico-social y las interacciones individuo-sociedad; sólo centran su interés en las decisiones que se toman. Como diría Moscovici, se ignora el proceso cognitivo y social que está detrás de las prácticas. $^{2}$

El consumo de drogas es un hecho y una práctica social sobre la que se toman decisiones, ¿cuál sería entonces el beneficio de diagramar los modelos de toma de decisiones sin entender el proceso cognitivo y social que los respalda? La región occidente de la República mexicana, a la que pertenece Jalisco, ocupa el segundo lugar en prevalencia de consumo alguna vez en la vida $(7.50 \%)$, por arriba de la media nacional $(5.27 \%)$. El comportamiento en el último año y en el último mes mantiene la misma relación. Las sustancias con mayor uso son la mariguana, con una prevalencia de $4.5 \%$, cocaína con $1.1 \%$ y los inhalables con $0.3 \%$. La mayor concentración es en hombres de entre 19 a 25 años de edad, y en mujeres de entre 26 a 34 años. ${ }^{16} \mathrm{El} 47 \%$ de la población tapatía se concentra en el intervalo de menores de 20 años de edad, lo que representa la población con mayor riesgo para el inicio del consumo de drogas. ${ }^{17,18}$

La familia, entendida como sistema, posee cualidades de autorregulación que le permiten emprender movimientos ante situaciones que la desestabilizan como el consumo de drogas de un hijo. Es sobre los padres en quienes recae la responsabilidad de protección, cuidado y educación de los hijos, y aun cuando una tarea del adolescente es desprenderse de las ligas familiares, la familia continúa siendo el contexto para su conducta. ${ }^{19}$

La investigación en familias de usuarios se ha centrado en caracterizarlas. Se ha asociado el consumo con estilos de comunicación defectuosa y triangulada, ${ }^{20}$ la existencia de familias multiproblemáticas, ${ }^{21}$ conflictos maritales, falta de acuerdos comunes, ${ }^{20}$ conflictos y pugnas entre padres e hijos, ${ }^{20,21}$ la existencia de alianzas y coaliciones intergeneracionales, ${ }^{22-24}$ la existencia de violencia intrafamiliar, ${ }^{23,24}$ patrones familiares repetitivos que perpetúan y mantienen la adicción, como por ejemplo, la existencia de un miembro que usa drogas ${ }^{25}$ el ambiente ${ }^{20}$ y la atmósfera familiar en la que se priva de manifestaciones de afecto y reconocimiento a cada uno de los integrantes, ${ }^{22}$ patrones ineficaces y estereotipados para resolver con las mismas estrategias cualquier problema, ${ }^{20,21}$ la baja satisfacción familiar, ${ }^{22,26}$ la percepción familiar tendiente a aspectos negativos, ${ }^{27-}$ ${ }^{29}$ la ruptura familiar ${ }^{30}$ y la práctica religiosa. ${ }^{31}$

Se ha descuidado el estudio de qué estrategias utiliza la familia para enfrentar la adicción y qué procesos cognitivos las respaldan. Desde esta perspectiva 
teórica, el objetivo del estudio fue explicar cómo y sobre qué representación social, los padres de adolescentes usuarios de drogas construyeron el modelo de toma de decisiones, de manera retrospectiva desde que advirtieron el consumo de drogas y hasta que acudieron a tratamiento a los Centros de Integración Juvenil (CIJ) en la ciudad de Guadalajara, Jalisco, México.

\section{Material y métodos}

Se trata de un estudio cualitativo de tipo analítico-interpretativo, de tres fases transversales. ${ }^{32}$ La población en estudio fueron 60 padres (78\% mamás, $11 \%$ papás, $11 \%$ parejas; 42 años de edad en promedio; 7.8 años de escolaridad; residentes en colonias urbano-marginales) que acompañaban a sus hijos adolescentes usuarios de drogas ilegales (56\% hombres, $44 \%$ mujeres) a tratamiento a CIJ en Guadalajara, Jalisco, México, durante 2002. La muestra fue calculada según el teorema central del límite ${ }^{33}$ y su selección fue aleatoria de tipo sistemático.

En la primera fase se utilizó una técnica de asociación libre ${ }^{6,34}$ mediante términos inductores y entrevistas de contexto a través de una guía que exploró el ambiente familiar y del barrio. Los términos inductores exploraron la imagen de la droga, del usuario, las creencias del ingreso a las drogas y los intentos de solución emprendidos. Para el análisis se calculó un listado de los descriptores asociados por el orden en que fueron mencionados, frecuencia y peso cultural ${ }^{35}$ (correlación entre frecuencia y orden de mención), de acuerdo con el software Anthropac 4.9. ${ }^{32}$ Las entrevistas se transcribieron en Atlas $\mathrm{Ti}^{36}$ para su lectura y relectura, con el fin de identificar códigos temáticos y crear familias de códigos. Por último, se realizó el análisis y su interpretación. En esta fase se plantearon premisas de cómo se objetivaba la RS. ${ }^{35}$

En la segunda fase, la información fue clasificada y jerarquizada por los participantes a través de cuestionarios de caracterización ${ }^{6}$ para identificar cómo los descriptores producidos se entrelazaban entre sí y plantear premisas del anclaje del modelo de toma de decisiones ${ }^{35}$ a rastrear en las entrevistas a profundidad.

El objetivo de la última fase fue la elaboración gráfica del modelo de toma de decisiones para identificar sobre qué RS se había construido, cómo había orientado las decisiones de los padres desde una dimensión retrospectiva a partir de que tomaron conocimiento del consumo de drogas del hijo(a) y hasta que habían llegado al CIJ. Para la recolección de la información se utilizaron entrevistas individuales a profundidad. En promedio, se utilizaron tres sesiones de dos horas cada una y fueron audiograbadas con previa autorización. El análisis fue el mismo que en las entrevistas de contexto.

En las tres fases, las medidas éticas fueron solicitar el consentimiento informado y salvaguardar la identidad de los participantes al modificar sus nombres con pseudónimos. Hubo dos casos en los que fue necesario referir a las madres a tratamiento por trastornos afectivos, se les dio seguimiento y no fue posible que continuaran participando. En este artículo se presentan los resultados generados a partir de la tercera fase.

\section{Resultados}

La representación sobre la que se construyó el modelo de toma de decisiones fue la oferta de un mundo mejor, organizada en tres momentos: a) descubriendo la adicción, b) la permanencia en las drogas y c) la retirada (figura 1).

\section{Descubriendo la adicción}

El modelo inició cuando las madres descubrieron la adicción de su hijo(a) ante un hecho que lo hizo evidente. Los padres (como nos referiremos a ambos) depositaron en los hijos expectativas que no incluían el consumo de drogas, aun cuando $70 \%$ reportó que el abuelo, el papá o los tíos del adolescente tenían un consumo problemático de alcohol y $67 \%$ de drogas. Este conjunto de expectativas, esperanzas y deseos hacia los hijos encarnó la representación que ha sido denominada la oferta de un mundo mejor, con la esperanza de que ellos vivieran una situación más favorable.

Augusta, mamá de Mariano: "yo esperaba que mi hijo algún día estudiara y tuviera una vida para adelante, no como yo la tuve, porque yo me estanqué. Yo no quería que se estancara, yo quería que fuera mejor que yo, para que el día de mañana no sufra como yo he sufrido, que tuviera una forma de vivir mejor".

Creían que por haber vivido los problemas del consumo familiar aprenderían y evitarían "caer en el mismo error". Sin embargo, el aprendizaje fue opuesto; las figuras masculinas más importantes modelaron conductas de aceptación y tolerancia al consumo. Cuando se enteraron del consumo del hijo, se hizo evidente el rechazo de ese mundo mejor y la existencia de un mundo diferente. La reacción de los padres no pudo ser otra que la decepción moral y emocional, porque se dieron cuenta que ese conjunto de expectativas, esperanzas y deseos, no sólo no se cumpliría, sino que 
1 Descubriendo la adicción

¿El hijo(a) reconoce su consumo ante un hecho que lo hace evidente?

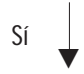

No

Decepción emocional de los padres y búsqueda de culpables, cuestionamiento de la parentalidad

Búsqueda de explicaciones que justifiquen el consumo

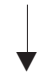

Primeros movimientos de la familia: involucramiento del padre y juramento del hijo. Rechazo a pedir ayuda porque querer es poder

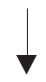

¿El padre tiene consumo problemático de alcohol o drogas?

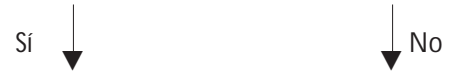

\section{Mayor comprensión Impone su autoridad}

Lo jura y se jura Lo jura

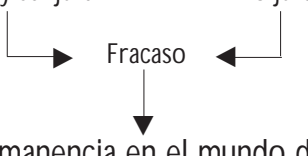

2 La permanencia en el mundo de las drogas Etapa prolongada

Inconsistencia en la parentalidad: amor-comprensión/castigos severos

Hacen más de lo mismo por la creencia querer es poder

Se activan viejos y surgen nuevos problemas: la destrucción familiar

¿La confrontación con el mundo real?

Se reconoce a la adicción como un problema que amerita ayuda por el síndrome de abstinencia del hijo y la incapacidad de controlarlo
No
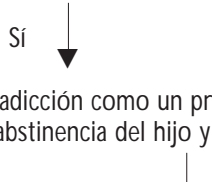

* DIF: Sistema N acional para el Desar rollo Integral de la Familia

₹ CIJ: Centros de Integración Juvenil

\section{Figura 1. Modelo de toma de decisiones de padres ante el consumo de drogas de sus hijos, basado en la REPRESENTACIÓN SOCIAL LA OFERTA DE UN MUNDO MEJOR}

además, desde su concepción, les esperaba una vida peor a la de ellos. De manera adicional, tuvieron la sensación de haber fracasado en la tarea de guiar al hijo hacia ese mundo.

Romeo, papá de Crispin: “ ¿quién quiere tener un hijo así?, a uno le duele ver la manera en cómo el hijo se está consumiendo, a veces uno ve muchachos drogadictos que andan por la calle, inhalando sus bolsitas y comparé a mi hijo con uno de ellos ... yo no quiero eso para él".

Si alguien era culpable, era la mamá. Primero, porque culturalmente su rol ha sido definido como el de cuidadora y educadora de los hijos, ${ }^{37}$ y segundo, porque como algunos papás mexicanos, éstos ocupaban un rol periférico. ${ }^{37} \mathrm{El}$ rol de proveedor no exige un cuidado afectivo y cercano, entonces, el descuido era de la mamá, quien fue percibida como ineficiente e incapaz. Esta imagen de la madre ineficiente y culpable fue compartida y aceptada por ellas mismas. Esta aceptación se observó de manera más consensuada en el caso de las mamás de usuarias mujeres, pues tradicionalmente se espera que ellas estén más vinculadas a su familia y se preparen para el oficio de madre. En cambio, en el caso de los hombres, hubo divergencias pues algunas no aceptaban ser las responsables y la culpa fue atribuida a las "malas compañías", en las que 
se develó a un mal amigo que presionó y a un hijo víctima en el que minimizaron su voluntad propia.

Una vez que los padres conocieron abiertamente el consumo del hijo(a), y que habían superado la crisis inmediata del impacto emocional, emprendieron dos movimientos: el involucramiento afectivo del papá y la búsqueda del juramento del hijo(a). Hasta esta etapa, el papá había asumido un rol periférico, pero como la madre resultó incompetente, el papá se vio obligado a adoptar este nuevo rol para imponer su autoridad como jefe de familia. El segundo movimiento correspondió a la búsqueda del juramento del hijo(a) para que dejara las drogas, que se encontró anclado en la creencia querer es poder. Por consecuencia, no estuvo disponible la opción de acudir a los servicios de salud. En parte, porque el querer es poder absorbió toda su creatividad para la búsqueda de otras estrategias fincando su eficacia en la propia voluntad, pero por otro lado, porque acudir a los servicios de salud significaba reconocer públicamente al hijo como adictovicioso, lo que se traduce en la exclusión de los grupos sociales. Al no exponerlo a ese reconocimiento, no se posibilitó la estigmatización social y se dio cobijo emocional.

Entonces, el juramento del hijo(a) era la opción más viable para resolver el problema, aunque no funcionó. Se observó que en el caso de los papás con antecedentes de consumo hubo un mayor entendimiento, comprensión y menos reproches hacia el hijo(a). En caso contrario, había menor tolerancia, menor comprensión y más reproches.

Esta crisis como resultado de la primera etapa, terapéuticamente hablando, fue paralizante por la resonancia emocional que causó y, por consecuencia, inmovilizó la capacidad creativa para emprender otros mecanismos de afrontamiento.

Los tiempos de esta etapa oscilaron entre 6 y 12 meses; sin embargo, el tiempo no fue lo determinante para transitar a la siguiente etapa sino el tipo de movimientos realizados; el ejercicio parental inconsistente y el reconocimiento de necesitar ayuda.

\section{La permanencia en el mundo de las drogas}

Como ya se conocía abiertamente el consumo, el adolescente se preocupaba menos por las condiciones en que llegaba a casa, lo que ocasionó mayores conflictos. Esto hizo evidente que la estrategia emprendida (el juramento) no había dado los resultados esperados, pues no sólo no había dejado de consumir, sino que "se había descarado". Esta situación generó nuevas medidas como alternar la parentalidad entre amorcomprensión y castigos severos-rechazo.
Sabina, mamá de Leocadia: "cuando ya estuvo así a lo descarado, de plano faltó a dormir, su papá ya le había pegado, ya habíamos hablado con ella, nos hacía creer que se portaba bien y no asistía a clases, llegó un día en que hacía lo que se le pegaba su gana".

Jurar al hijo y ejercer una parentalidad inconsistente fueron estrategias más de lo mismo; es decir, prácticas aparentemente diferentes ancladas en una misma creencia, que sólo complicaron más el problema, pues evolucionó en el tiempo sin el empleo de una solución efectiva. Los padres tenían la certeza de que bastaba querer, entonces, a veces, eran cariñosos para mover con afecto la voluntad, los dejaban hacer lo que ellos querían, los buscaban cuando no llegaban, no les negaban nada; pero cuando veían que no les funcionaba, empleaban castigos como retirarles a los amigos, no dejarlos salir, no darles dinero, ponerlos a trabajar, e incluso golpearlos, dejarlos sin comer y no dejarlos entrar a la casa.

Abigail, mamá de Ruperto: "yo la verdad, ya no sé ni cómo manejarlo, ya no sé qué hacer, a veces digo lo voy a castigar y no le voy a echar lonche, a veces, hasta le llevo el chocomilk hasta la cama, y luego digo, ¿para qué sirven tantos esfuerzos? ... Mi esposo le ha pegado, a veces, lo maltrata, habla con él y nada, él me dice: 'Amá déjeme aprender de mis errores porque si usted me lo dice, no se la creo, déjeme a mí" ".

Estos movimientos más de lo mismo, crearon un primer círculo vicioso que detuvo la evolución natural del problema al paralizar el proceso creativo de la búsqueda de solución del conflicto, pues aunque reconocieron que era un problema que ameritaba atención no percibían que fuera lo suficientemente grave como para buscar alternativas fuera de ellos mismos, y prosiguió su esfuerzo mediante acciones de rutina. Esta fue la etapa más prolongada y desgastante para padres y adolescentes, por la cantidad de recursos psico-emocionales y afectivos invertidos. Su duración osciló entre 12 meses y 6 años.

Cuando nuevamente estos intentos no surtieron los efectos esperados se instauraron, con mayor intensidad en la madre, sensaciones de frustración, tensión y desaliento que provocaron una desactivación del nivel óptimo de motivación que paralizó el segundo proceso natural de evolución del problema: el proceso cooperativo. El papá, volvió a la periferia para disminuir la tensión y la angustia generada, y la madre, se dejó envolver por la rutina cotidiana de tolerancia. Estas nuevas conductas perfilaron un ambiente fami- 
liar y estados emotivos poco óptimos para la familia, el alto grado de estrés y los nuevos conflictos que aparecieron, caracterizaron lo que ellos llamaron la destrucción familiar. Desde el sentido común, que opera bajo reglas ingenuas y reduccionistas, la destrucción familiar fue explicada por una causa: el consumo de drogas. Desde este razonamiento, una vez que el hijo(a) dejara de consumir, la familia volvería a ser lo que fue.

El último elemento que caracterizó a este primer círculo vicioso fue la confrontación con el mundo real, que se hizo presente con el síndrome de abstinencia, cuando el hijo no dejó las drogas después de transcurrido un tiempo que ellos consideraron como natural. Ante el síndrome de abstinencia, que hasta este momento había sido ignorado, los padres se dieron cuenta que consumía por necesidad, que su cuerpo se lo pedía y aceptaron que el problema era superior a la voluntad y que no bastaba con querer.

Con este choque de creencias y ante la impotencia, sufrimiento, cansancio e incapacidad para controlar el consumo del hijo(a), se consideró la posibilidad de buscar ayuda fuera de la familia. Esta nueva concepción implicaba reconocer socialmente al hijo como adicto, pero se re-definió como un adicto-enfermo, que implicaba su aceptación y la posibilidad de darle cobijo emocional, pues a los enfermos no se les excluye de los grupos sociales, se les quiere y atiende para que se recuperen. Desde este razonamiento surge la concepción del ingreso a las drogas por error. Un error que sería enmendado por la rehabilitación. Este nuevo razonamiento condujo a un nuevo movimiento: ofrecerle al hijo buscar ayuda especializada, con la promesa de encontrar un mundo liberado de las drogas. Sin embargo, a pesar de considerar esta posibilidad, en tanto el hijo(a) no aceptó la ayuda, se formó un segundo círculo vicioso que detuvo el debut en los servicios de salud, pues operaba la creencia "mi consumo no es problema".

\section{La retirada}

Esta tercera fase inició cuando finalmente el adolescente aceptó que su consumo era problemático. La madre buscó ayuda en amistades, familiares y personas conocidas que habían vivido un problema similar y en opciones culturalmente reconocidas de ayuda, como sacerdotes, líderes de otras religiones, maestros, psicólogos, drogadictos anónimos, CIJ, medios de comunicación como programas de televisión y radio y consultas médicas. Esta búsqueda de servicios de salud se caracterizó por el pensamiento de la última esperanza, en el que depositaron la expectativa de que el síntoma iba a desaparecer una vez iniciado el tratamiento. Se observó a una madre con nuevas motivaciones y una mayor aceptación del hijo(a) como usuario bajo la concepción de enfermo con posibilidades de rehabilitación.

Sin embargo, sus expectativas se vieron frustradas porque conforme avanzaban en las citas no observaron cambios; el consumo y las conductas asociadas seguían, lo que provocó sensaciones de desesperanza y mayor frustración. En esta última etapa, el pensamiento de los padres era pesimista al definir a la adicción como un problema sin salida. Se observó a una madre agotada, depresiva, con poco aliento para continuar realizando inversiones, y a un papá que se mantenía en la periferia. Terapéuticamente hablando, una familia vulnerable para un segundo brote o la aparición de otras conductas autodestructivas en el hijo(a) activo(a) o en algún otro miembro de la familia.

\section{Discusión}

Moscovici ${ }^{2}$ apuntó que las representaciones sociales se erigen como modelos de interpretación que guían las tomas de decisión. En este sentido, el estudio representa un modelo explicativo de la adicción, desde el sentido común de estos padres, y cómo de estas formas de pensamiento social se desprenden acciones que no son arbitrarias, sino producto de razonamientos con los que afrontaron la adicción del hijo(a) con anclajes eminentemente culturales -como las creencias socialmente compartidas-e idiosincrásicas -como las afectividades.

Este modelo, que podría denominarse modelo de sentido común de la adicción, que explica cómo se ingresa a las drogas, no deja de ser reduccionista -como todo sentido común-. Seguramente la adicción va más allá de intentar materializar un mundo seguro para los hijos; sin embargo, estos padres están utilizando dichos parámetros para explicar la realidad que viven, ¿por qué habrían de cotejar sus ideas con el parámetro de un pensamiento científico? Lo único con lo que cuentan son con sus creencias, sentimientos, actitudes y afectividades, y con esto explican, verifican, distinguen $\mathrm{y}$ toman decisiones acerca de lo que les acontece.

Aun cuando el modelo de toma de decisiones se encuentra organizado en tres fases diferentes la representación que las orientó fue la que hemos denominado la oferta de un mundo mejor, que surge de una condición problemática en la familia. Por un lado, se tolera el consumo de drogas en figuras masculinas con jerarquía, y por otro, se espera que el adolescente aprenda de los errores. Es a lo que Tomori ${ }^{25}$ se refería con la existencia de patrones familiares que perpetúan y man- 
tienen la adicción, o lo que la terapia centrada en soluciones $^{9,14}$ denomina la pauta adictiva que conecta a una generación con otra.

Desde el marco de las RS agregaríamos que esta práctica se mantiene además por la existencia de una creencia socialmente compartida: querer es poder, que resulta de la concepción "los vicios se dejan con voluntad". La adicción como vicio frena el uso de los servicios de salud, al considerar que pueden ser excluidos de los grupos sociales. La adicción como enfermedad, en cambio, posibilita su inclusión. Esta modificación cultural que ocurre en la última fase es lo que posibilita la búsqueda de ayuda fuera de la familia.

De manera adicional a este componente idiosincrásico de la redefinición de la adicción, creemos que se encuentra involucrado otro componente eminentemente cultural: la imagen y función socialmente construida de los servicios. La población utiliza los servicios de salud mental ante la percepción de gravedad, pues la cuestión de la aceptación social parece ser un mediador. ${ }^{38}$ Se ha encontrado que aunque la población tenga información acerca de sus padecimientos y acepta la cercanía con los enfermos, la búsqueda de atención se ha relacionado con creencias, actitudes e intenciones y se utilizan cuando ya hay una larga evolución y se ha vuelto crítico su manejo. ${ }^{38}$ La Encuesta Nacional de Epidemiología Psiquiátrica reveló que sólo 1 de cada 10 personas con algún trastorno recibió atención. ${ }^{39}$

Estos hallazgos muestran la problemática que enfrentan los servicios de salud. La población parece tener buena disposición para buscar y recibir ayuda, sabe a dónde acudir, ${ }^{40}$ pero parece que acude hasta el momento en que se da cuenta que con sus medios no podrá resolverlos. Los hallazgos revelan que en la adicción parece similar. Desde esta perspectiva, la imagen y función atribuidas a los servicios de salud no favorecen su uso.

Por otro lado, algunos estudios han propuesto que la adicción brota como consecuencia de disfunciones familiares y se les ha conceptualizado como familias multiproblemáticas. ${ }^{21}$ En este estudio, en efecto, observamos algunas disfunciones, pero las condiciones generadoras de estrés no sólo se ubican en el medio familiar. La representación del mundo mejor es eminentemente social, pues se encuentra anclado en la figura de ser buenos padres ${ }^{35}$ al intentar materializar un mundo mejor para los hijos y se esfuerzan como si el propósito dependiera sólo de la voluntad al tratar a toda costa de materializarlo sólo al interior de la familia al hacer un recorte con el resto del mundo, ignorando, por ejemplo, la influencia del contexto social. En parte, este pensamiento subyace en dos creencias: a) la cuestión de la invulnerabilidad al creer que "a mí no me va a pasar, mi hijo no va a ser un adicto", en todo caso, creen que va a aprender de los errores, a pesar del modelamiento que ocurre en su interior, y b) la creencia querer es poder, en donde pareciera que basta el deseo de que el hijo deje las drogas para resolver su consumo.

Desde este marco, las disfunciones no son movimientos arbitrarios, sino productos de razonamientos de sentido común que cumplen una función. Se ha señalado que la familia emprende patrones ineficaces y estereotipados para resolver con las mismas estrategias sus problemas, lo que mantiene la adicción. ${ }^{20,21}$ La familia actúa así porque necesita buscar un equilibrio para sobrevivir emocionalmente, y entonces, se vuelve tolerante e ineficiente, pero esta ineficacia es tal porque los padres no están en condiciones de hacer algo más; el impacto y el choque emocional de tener un hijo adicto no les permite hacer nada más. Nadie está preparado para tener un hijo(a) adicto. Pero por otro lado, se tiene presente la presión cultural del contexto inmediato, que tiende a rechazar y a segregar a los usuarios, ${ }^{41}$ entonces, la familia se perfila como el único ambiente disponible que puede protegerlos ante esa amenaza, lo que crea un círculo vicioso que frena la proyección de una solución exitosa.

De ello resulta una tipología de padres al interior: los sobreprotectores y los periféricos. Como dice Mar$\mathrm{kova}^{42}$ se trata de una relación dialógica, que desde el punto de vista sistémico, cumple una función compensatoria de género, ${ }^{43}$ mientras el padre se comporta de manera periférica porque su rol le exige ser un buen proveedor, la madre compensa su función con estrategias de tipo sobreprotector para guiar al hijo a ese mundo prometido. Sin embargo, cuando se hace obvio el fracaso de la madre para guiar al hijo a ese mundo, el padre retoma su autoridad como jefe de familia para tratar de resolver, desde otra estrategia aparentemente diferente, su consumo.

Entonces, la adicción no puede ser explicada sólo en términos de la disfunción familiar, como lo sugieren los estudios, e incluso el marco teórico de la terapia familiar. Los hallazgos sugieren que las familias entrevistadas son altamente sensibles a consumir drogas, en parte, porque ha sido un comportamiento modelado por su generación previa, pero también porque es una forma de afrontar el estrés y la ansiedad que genera el tratar a toda costa de materializar un mundo mejor para los hijos bajo circunstancias contextuales adversas.

La representación social que orienta la toma de decisiones en los padres no es autónoma, porque como dice Moscovici, ${ }^{2}$ la representación social condensa una 
reflexión colectiva diversificada y difusa. De ahí que sean multifacéticas. La representación un mundo mejor, está vinculada a lo que es, o mejor dicho, lo que debe ser, una familia, en términos normativos, lo que se espera y lo que no se espera de un hijo. Como dice Flores ${ }^{43}$ al haber un sistema central que determina el sentido de una RS, pueden coexistir diferentes significados que expliquen un mismo objeto de representación por un grupo homogéneo. La población en estudio explicó la adicción en función de lo que se espera de los hijos, lo que la vincula a otra representación social.

En síntesis, se trata de un estudio que rastreó, en retrospectiva, las decisiones emprendidas desde el descubrimiento de la adicción hasta cuando acuden a los servicios y los elementos cognitivos que las respaldan. Se cuenta con un modelo de toma de decisiones construido por una población finita en el cual advertimos rasgos socialmente compartidos, sin embargo, ¿este modelo opera de manera similar en otras poblaciones o es eminentemente de carácter idiosincrásico?, ¿será posible predecir, ahora en prospectiva, tales decisiones? Creemos que estas preguntas podrían ser contestadas utilizando la metodología de modelos de decisiones de árboles etnográficos. ${ }^{44}$

Por último, sería oportuno trabajar en grupos psico-educativos con los padres, para atender su estado emocional, fortalecer habilidades parentales, orientarlos sobre el proceso y la conducta adictiva y trabajar en las creencias que obstaculizan el curso del conflicto. Se ha reportado que estos programas proveen modelos de roles para la conducta en salud ${ }^{45}$ impactan positivamente en la reducción de barreras cognitivas, en la utilización adecuada de respuestas parentales, en la moderación del consumo $\mathrm{o}^{46,47}$ e indican que se promueve una red de apoyo y cohesión que a largo plazo es la más importante para mantenerse sin consumo. ${ }^{48}$

\section{Referencias}

1. Moscovici S. La psychanalyse son image et son public. Paris, France: Presses Universitaires de France; 1961.

2. Moscovici S. El psicoanálisis, su imagen y su público. BuenosAires, Argentina: Huemul; 1979.

3. Di Giacomo JP.Teoría y métodos de análisis de las representaciones sociales. En: Páez D, ed. Pensamiento, individuo y sociedad: cognición social y presentación social. Madrid: Fundamento; 1987:78-85.

4. Páez D. Características, funciones y proceso de representaciones sociales. En: Páez D, ed. Pensamiento, individuo y sociedad: cognición social y representación social. Madrid: Fundamento; 1987:37-48. 5. Jodelet $D$. Representaciones sociales: contribución a un saber sociocultural sin fronteras. En: Jodelet D, G uerrero A, ed. D evelando la cultura. Estudios en representaciones sociales. México, DF: UN AM; 2000:7-30.

6. Abric JC. Prácticas sociales y representaciones. México, D F: Ed.

Coyoacán;2001:75-96.

7. Kuhn T. La estructura de las revoluciones científicas. México, DF:

Fondo de Cultura Económica; 1962.

8. Reiss D.The family's construction of reality. C ambridge: University Press; 1981.

9. Simon F, Stierlin H,W ynne L.Vocabulario de terapia familiar. Barcelona, España: G edisa; 1997.

10. De Shazer S. Claves para la solución en terapia breve. Barcelona, España: Paidós; 1995.

11. De Shazer S. Patterns of brief family therapy:An ecosystemic approach. N ew York (N Y): Guilford Press; 1982.

12. Kantor D, Lehr W. Inside the family:Toward a theory of family process. San Francisco, EU: Jossey-Bass; 1975.

13. Keeney B. Ecosystemic epistemology: An alternative paradigm for diagnosis. Fam Process 1979;18:117-129.

14. Landau J.Therapy with families in cultural transition. En: McG oldrick M, Pearce JK, Giordano J, ed. Ethnicity and family therapy. Second edition. N ew York (N Y): G uilford Press; 1982:552-572.

15. Lewin K. D inámica de la personalidad. Madrid: Morata; 1973. 16. Dirección General de Epidemiología, Secretaría de Salud. Segunda Encuesta N acional de Adicciones 1993. México, DF: SSA; 1994. 17.Villatoro J, Medina-Mora ME, López E, Juárez F, Rivera E, Fleiz C. La dependencia y los problemas asociados al consumo de drogas en México. Resultados de la Encuesta $\mathrm{N}$ acional de Adicciones. Salud Mental 1996;19:1-6.

18. Medina-Mora ME,Villatoro J, López E, Berenzon S, Carreño S, Juárez F. Los factores que se relacionan con el inicio, el uso continuado y el abuso de sustancias psicoactivas en adolescentes mexicanos. Gac Med Mex 1995;131:383-387.

$19 \mathrm{~N}$ uño B. La familia con adolescentes. En: N uño B, Madrigal E, ed. 0 rientaciones educativas para padres de adolescentes. México, DF: Universidad de Guadalajara; 1999:61-75.

20. Klein K, Forehand R,Armistead L, Long P. D elinquency during the transition to early adulthood: Family and parenting predictors from early adolescence. Adolescence 1997;32:61-80.

21. Sokol J, D unham R, Zimmerman R. Family structure versus parental attachment in controlling adolescence deviant behavior:A social control model. Adolescence 1997;32:199-215.

22.Yeh L, Hedgespeth J. A multiple case study comparison of normal private preparatory school and substance abusing/mood disordered adolescents and their families. Adolescence 1995;30:413-428.

23. Straus M, Kaufman G. Corporal punishment of adolescents by parents: $A$ risk factor in the epidemiology of depression, suicide, alcohol abuse, child abuse, and wife beating.A dolescence 1994;29:543-561.

24. Graham N . The influence of predictors on adolescent drug use. An examination of individual effects. Youth Soc 1996;28:215-235.

25. Tomori M. Personality characteristics of adolescents with alcoholic parents. Adolescence 1994;29:949-959.

26. Choquet M, Kovess V, Poutignat, N . Suicidal thoughts among adolescents: An intercultural approach. Adolescence 1993;28:649-653. 27. Anderson A, H enry C . Family system characteristics and parental behaviors as predictors of adolescent substance use. Adolescence 1994:28:405-420.

28. Foxcroft D, Lowe G.Adolescent drinking, smoking and other substance use involvement: Links with perceived family life. J Adolesc 1995;18:159-177.

29. D enton R, Kampfe H.The relationship between family variables and adolescent substance abuse: $A$ literature review. Adolescence 1994:29:475-495.

30. Hagell A, N ewburn T. Family and social contexts of adolescent re-offenders. J Adolesc 1996;19:5-18. 
31. Foshee A, Hollinger R. Maternal religiosity, adolescent social bonding, and adolescent alcohol use. J Early Adolesc 1996;16:451-468. 32. Borgatti S.Anthropac 4.9 Software. N atick (MA).A nalytic Technologies; 1996.

33. Canavos GC. Probabilidad y estadística. A plicación y métodos. México, DF: McGraw-Hill; 1998.

34. Flores F, Díaz JA. N ormalidad y anormalidad: esquemas dicotómicos de la representación social en un grupo de profesionales de la salud mental. Polis 2000:247-263.

35. N uño B. De la representación a la acción: modelos de toma de decisiones con los que intentan resolver el consumo de drogas ilegales adolescentes consumidores y sus padres [Tesis para obtener el grado de doctora en psicología social]. México, DF: Facultad de Psicología, UNAM; 2004

36. Muhr T.Atlas Ti. Scientific software development. Berlin, 1998.

37. Ramírez S. El mexicano, psicología de sus motivaciones. México; DF: Grijalbo; 1978.

38. G arcía-Silberman S. Un modelo explicativo de la conducta hacia la enfermedad mental. Salud Publica Mex 2002;44:289-296.

39 Medina-Mora ME, Borges G, Lara-Muñoz C, Benjet C, Blanco-Jaimes J, Fleiz-Bautista $C$ et al. Prevalencia de trastornos mentales y uso de servicios: resultados de la Encuesta $\mathrm{N}$ acional de Epidemiología Psiquiátrica en México. Salud Mental 2003;26(4):1-16.
40 García-Silberman S. N ecesidades de atención y utilización de servicios de salud mental. Salud Mental 1997;20(supl 2):S34-S46. 41. Goffman E. Estigma. La identidad deteriorada. Buenos Aires, Argentina:Amorrortu; 1995.

42. Marková I.The individual and the community:A post-communist perspective. J Appl Soc Psychol 1997;7:3-17.

43. Flores F. Psicología social y género. El sexo como objeto de representación social. México, DF: McG raw-Hill; 2001.

44. G ladwin CH. Etnographic decision tree modeling. Q ualitative research methods. Thousand 0 aks, EU: SAGE; 1989.

45. Perry CL. Community wide health promotion and drug abuse prevention. J Sch Health 1986:56;36-42.

46. Johnson K, Bryant D, Strader T, Bucholtz G, Berbaum M, Collins D et al. Reducing alcohol and other drug use by strengthening community, family, and youth resiliency: An evaluation of the creating lasting connections program. J Adolesc Res 1996;11:36-67.

47. Pierre TL, Mark MM, Kaltreider DL,Aikin HL. Involving parents of high-risk youth in drug prevention:A three-year longitudinal study in boys $\&$ girls clubs. J Early Adolesc 1997;17:21-50.

48. Barber JG. Social work with addictions. G reat Britain, England: Macmillan Press; 1995 